九州大学学術情報リポジトリ

Kyushu University Institutional Repository

\title{
ON SEQUENTIAL ESTIMATION OF THE REGRESSION FUNCTION
}

Samanta, M

Department of Statistics, The University of Manitoba

https://doi.org/10.5109/13352

出版情報: Bulletin of informatics and cybernetics. 21 (1/2), pp.19-27, 1984-03. Research Association of Statistical Sciences

バージョン :

権利関係 : 


\title{
ON SEQUENTIAL ESTIMATION OF THE REGRESSION FUNCTION*
}

\author{
By
}

\section{SAMANTA**}

\section{Introduction and Summary}

Let $(X, Y)$ be a two dimensional random variable having a joint density function $f$ and let $g$ be the marginal density function of $X$. We assume that $E_{f} Y$ is finite and define the regression function $m(x)$ (for regression of $Y$ on $X$ ) by $m(x)=E[Y \mid X=x]$. Nadaraya [3] and Watson [7] and Schuster [6] have studied the asymptotic properties of the estimate $\hat{m}_{n}(x)$ of $m(x)$ defined by

$$
\hat{m}_{n}(x)=\frac{\sum_{i=1}^{n} Y_{i} K\left(\frac{x-X_{i}}{h_{n}}\right)}{\sum_{i=1}^{n} K\left(\frac{x-X_{i}}{h_{n}}\right)}
$$

where $K(u)$ is a probability density function on $(-\infty,+\infty),\left\{h_{n}\right\}$ is a monotonically decreasing sequence of positive numbers converging to zero and $\left(X_{1}, Y_{1}\right),\left(X_{2}, Y_{2}\right), \cdots$, $\left(X_{n}, Y_{n}\right)$ are $n$ independent observations of $(X, Y)$.

In many practical situations the number of observations $N_{t}$ which we observe in time $(0, t]$ is a random variable. We call $N_{t}$ a stopping random variable. We assume that $\left(X_{1}, Y_{1}\right),\left(X_{2}, Y_{2}\right), \cdots$ are independent observations of $(X, Y)$ and need not be independent of the random variable $N_{t}$. In this paper we propose an estimate $m_{N_{t}}(x)$ of the regression function $m(x)$ based on $\left(X_{1}, Y_{1}\right),\left(X_{2}, Y_{2}\right), \cdots,\left(X_{v_{t}}, Y_{v_{t}}\right)$ and given by

$$
m_{v_{t}}(x)=\frac{\sum_{i=1}^{N_{t}} \frac{Y_{i}}{h_{i}} K\left(\frac{x-X_{i}}{h_{i}}\right)}{\sum_{i=1}^{N_{t}}-\frac{1}{h_{i}} K\left(\frac{x-X_{i}}{h_{i}}\right)}
$$

We note that the expression for the estimate $m_{N_{t}}(x)$ is motivated by the recursive type of estimate of a probability density function first proposed by Yamato [8]. Suppose $x_{1}, x_{2}, \cdots, x_{l}$ are $l$ distinct points. We have shown that under certain regularity conditions $\left(N_{t} h_{N_{t}}\right)^{1 / 2}\left\{m_{N_{t}}\left(x_{1}\right)-m\left(x_{1}\right), \cdots, m_{N_{t}}\left(x_{l}\right)-m\left(x_{l}\right)\right\}$ is asymptotically normally distributed with mean vector 0 and diagonal covariance matrix $C=\left[c_{i j}\right]$ with $c_{i i}=$ $\frac{\operatorname{Var}\left[Y \mid X=x_{i}\right]}{g\left(x_{i}\right)} \nu \int_{-\infty}^{\infty} K^{2}(u) d u$ where $\nu(\nu<1)$ is as defined in the next section. For simplicity we have proved the theorem for the special case $l=2$. The method of proof remains valid in the more general case. The theorem can be regarded as the appropriate

* Research partially supported by a Natural Science and Enging Research Council Grant of Canada.

** Department of Statistics, The University of Manitoba, Winnipeg, Manitoba, R3t 2n2 Canada. 
extension of the earlier result due to Schuster [6].

\section{Main Result}

We assume that the probability density function $K$ and the sequence $\left\{h_{n}\right\}$ are chosen to satisfy the following conditions :

(i) $K(u)$ and $|u K(u)|$ are bounded

(ii) $\int_{-\infty}^{\infty} u K(u) d u=0$

(iii) $\int_{-\infty}^{\infty} u^{2} K(u) d u<\infty$

(iv) $\lim _{n \rightarrow \infty} \frac{1}{n} \sum_{j=1}^{n}\left(\frac{h_{n}}{h_{j}}\right)=\nu<1$

(v) $\frac{1}{n} \sum_{j=1}^{n} h_{j}^{2} \leqq C_{1} h_{\bar{n}}^{2} \quad\left(C_{1}>0\right), n=1,2,3, \cdots$

(vi) $\lim _{n \rightarrow \infty} n h_{n}^{3}==\infty$ and $\lim _{n \rightarrow \infty} n h_{n}^{5}=0$

REMARK 1 . If $K$ is the standard normal probability density function, then conditions (i), (ii) and (iii) are satisfied.

REMARK 2. If $h_{n}=n^{-\delta}, \frac{1}{5}<\delta<\frac{1}{3}$, then conditions (iv), (v) and (vi) are satisfied. (For a proof see [1], p. 26 and p. 46.)

We define the following:

$$
\begin{aligned}
& g(x)=\int_{-\infty}^{\infty} f(x, y) d y \\
& w(x)=\int_{-\infty}^{\infty} y f(x, y) d y \\
& v(x)=\int_{-\infty}^{\infty} y^{2} f(x, y) d y .
\end{aligned}
$$

Hence,

$$
\operatorname{Var}[Y \mid X=x]=\frac{v(x)}{g(x)}-\frac{w^{2}(x)}{g^{2}(x)} .
$$

Suppose $x_{1}$ and $x_{2}$ are two distinct points. We define for $i=1,2, \cdots, n$ and $s=1,2$

$$
\begin{aligned}
& U_{i}^{*}\left(x_{s}\right)=\frac{1}{h_{i}} K\left(\frac{x_{s}-X_{i}}{h_{i}}\right) \\
& V_{i}^{*}\left(x_{s}\right)=Y_{i} U_{i}^{*}\left(x_{s}\right) \\
& U_{i}\left(x_{s}\right)=h_{n}^{1 / 2}\left\{U_{i}^{*}\left(x_{s}\right)-E U_{i}^{*}\left(x_{s}\right)\right\} \\
& V_{i}\left(x_{s}\right)=h_{n}^{1 / 2}\left\{V_{i}^{*}\left(x_{s}\right)-E V_{i}^{*}\left(x_{s}\right)\right\} \\
& \bar{U}_{n}\left(x_{s}\right)=\sum_{i=1}^{n} U_{i}\left(x_{s}\right)
\end{aligned}
$$




$$
\begin{aligned}
& \bar{V}_{n}\left(x_{s}\right)=\sum_{i=1}^{n} V_{i}\left(x_{s}\right) \\
& W_{i}=\left(U_{i}\left(x_{1}\right), V_{i}\left(x_{1}\right), U_{i}\left(x_{2}\right), V_{i}\left(x_{2}\right)\right)^{\prime} \\
& n^{1 / 2} Z_{n}=\left(\bar{U}_{n}\left(x_{1}\right), \bar{V}_{n}\left(x_{1}\right), \bar{U}_{n}\left(x_{2}\right), \bar{V}_{n}\left(x_{2}\right)\right)^{\prime} \\
& A=\nu \int_{-\infty}^{\infty} K^{2}(u) d u\left(\begin{array}{cccc}
g\left(x_{1}\right) & w\left(x_{1}\right) & 0 & 0 \\
w\left(x_{1}\right) & v\left(x_{1}\right) & 0 & 0 \\
0 & 0 & g\left(x_{2}\right) & w\left(x_{2}\right) \\
0 & 0 & w\left(x_{2}\right) & v\left(x_{2}\right)
\end{array}\right)
\end{aligned}
$$

Let $Z$ be a four variate normal random variable with mean vector 0 and covariance matrix $A$.

We now prove the following lemmas.

LEMMA 1. Suppose $K$ satisfies conditions (i) and (iii) and the sequence $\left\{h_{n}\right\}$ satisfies condition (iv). Let $g^{\prime}, w^{\prime}$ and $v^{\prime}$ exist and be bounded. Then the following results hold for $s=1,2$ and $r=1,2$.

(a) $\lim _{n \rightarrow \infty} \operatorname{Var}\left\{n^{-1 / 2} \bar{U}_{n}\left(x_{s}\right)\right\}=\nu g\left(x_{s}\right) \int_{-\infty}^{\infty} K^{2}(u) d u$

(b) $\lim _{n \rightarrow \infty} \operatorname{Var}\left\{n^{-1 / 2} \bar{V}_{n}\left(x_{s}\right)\right\}=\nu v\left(x_{s}\right) \int_{-\infty}^{\infty} K^{2}(u) d u$

(c) $\lim _{n \rightarrow \infty} \operatorname{Cov}\left\{n^{-1 / 2} \bar{U}_{n}\left(x_{s}\right), n^{-1 / 2} \bar{V}_{n}\left(x_{s}\right)\right\}=\nu w\left(x_{s}\right) \int_{-\infty}^{\infty} K^{2}(u) d u$

(d) $\lim _{n \rightarrow \infty} \operatorname{Cov}\left\{n^{-1 / 2} \bar{U}_{n}\left(x_{1}\right), n^{-1 / 2} \bar{U}_{n}\left(x_{2}\right)\right\}=0$

(e) $\lim _{n \rightarrow \infty} \operatorname{Cov}\left\{n^{-1 / 2} \bar{V}_{n}\left(x_{1}\right), n^{-1 / 2} \bar{V}_{n}\left(x_{2}\right)\right\}=0$

(f) $\lim _{n \rightarrow \infty} \operatorname{Cov}\left\{n^{-1 / 2} \bar{U}_{n}\left(x_{s}\right), n^{-1 / 2} \bar{V}_{n}\left(x_{r}\right)\right\}=0, \quad r \neq s$.

PROOF. We sketch the proof of part (a) and part (d) of the Lemma. The proof of the other parts are similar and will be omitted. To obtain part (a) we have

$$
\begin{aligned}
\operatorname{Var}\left\{n^{-1 / 2} \bar{U}_{n}\left(x_{s}\right)\right\}= & \frac{1}{n} \sum_{j=1}^{n}\left(\frac{h_{n}}{h_{j}}\right) \int_{-\infty}^{\infty}\{K(u)\}^{2} g\left(x_{s}-h_{j} u\right) d u \\
& -\frac{h_{n}}{n} \sum_{j=1}^{n}\left\{\int_{-\infty}^{\infty} K(u) g\left(x_{s}-h_{j} u\right) d u\right\}^{2} \\
= & \frac{1}{n} \sum_{j=1}^{n}\left(\frac{h_{n}}{h_{j}}\right)\left[g\left(x_{s}\right) \int_{-\infty}^{\infty}\{K(u)\}^{2} d u+O\left(h_{j}\right)\right] \\
& -\frac{h_{n}}{n} \sum_{j=1}^{n}\left[g\left(x_{s}\right)+O(h)\right]_{j} .
\end{aligned}
$$

Hence,

$$
\lim _{n \rightarrow \infty} \operatorname{Var}\left\{n^{-1 / 2} \bar{U}_{n}\left(x_{s}\right)\right\}=\nu g\left(x_{s}\right) \int_{-\infty}^{\infty} K^{2}(u) d u, \quad s=1,2 .
$$


To prove part (d) we have used the method similar to that of Schuster [6]:

$$
E\left[\frac{1}{h_{i}} K\left(\frac{x_{1}-X_{i}}{h_{i}}\right) K\left(\frac{x_{2}-X_{i}}{h_{i}}\right)\right]=O\left(h_{i}\right)
$$

Now,

$$
\begin{aligned}
\operatorname{Cov}\left\{n^{-1 / 2} \bar{L}_{n}\left(x_{1}\right), n^{-1 / 2} \bar{C}_{n}\left(x_{2}\right)\right\}= & \frac{1}{n} \sum_{i=1}^{n} E\left\{U_{i}\left(x_{1}\right) U_{i}\left(x_{2}\right)\right\} \\
= & \frac{1}{n} \sum_{i=1}^{n}\left[\frac{h_{n}}{h_{i}} E\left\{\frac{1}{h_{i}} K\left(\frac{x_{1}-X_{i}}{h_{i}}\right) K\left(\frac{x_{2}-X_{i}}{h_{i}}\right)\right\}\right. \\
& \left.-h_{n} E\left\{\frac{1}{h_{i}} K\left(\frac{x_{1}-X_{i}}{h_{i}}\right)\right\} E\left\{\frac{1}{h_{i}} K\left(\frac{x_{2}-X_{i}}{h_{i}}\right)\right\}\right] \\
= & \frac{1}{n} \sum_{i=1}^{n}\left[\frac{h_{n}}{h_{i}} \cdot O\left(h_{i}\right)-h_{n}\left\{g\left(x_{1}\right)+O\left(h_{i}\right)\right\}\left\{g\left(x_{2}\right)+O\left(h_{i}\right)\right\}\right] \\
= & O\left(h_{n}\right) .
\end{aligned}
$$

Hence,

$$
\lim _{n \rightarrow \infty} \operatorname{Cov}\left\{n^{-1 / 2} \bar{U}_{n}\left(x_{1}\right), n^{-1 / 2} \bar{U}_{n}\left(x_{2}\right)\right\}=0 .
$$

Let $C=\left(c_{1}, d_{1}, c_{2}, d_{2}\right)^{\prime}$ be any real vector in $R^{4}$.

Lemma 2. Suppose $K$ satisfies conditions (i) and (iii) and the sequence $\left\{h_{n}\right\}$ satisfies condition (iv) and $n h_{n}^{3} \rightarrow \infty$ as $n \rightarrow \infty$. Let $E_{f}|Y|^{3}$ be finite and let $g^{\prime}, w^{\prime}$ and $v^{\prime}$ exist and be bounded. If $g\left(x_{i}\right)>0$ for $i=1,2$, then $C^{\prime} Z_{n}$ converges in distribution to a normal random variable with mean 0 and variance $C^{\prime} A C$.

Proof. We shall establish the asymptotic normality of $C^{\prime} Z_{n}$ by showing that

$$
\lim _{n \rightarrow \infty}\left\{\frac{\sum_{i=1}^{n} E\left|C^{\prime} W_{i}\right|^{3}}{n^{3 / 2}\left(\operatorname{Var}\left(C^{\prime} Z_{n}\right)\right)^{3 / 2}}\right\}=0 \text {. (See [2], p. 275.) }
$$

Using Lemma 1, we have

$$
\begin{aligned}
\lim _{n \rightarrow \infty} \operatorname{Var}\left(C^{\prime} Z_{n}\right) & =\nu \int_{-\infty}^{\infty} K^{2}(u) d u\left[\sum_{s=1}^{2}\left\{c_{s}^{2} g\left(x_{s}\right)+d_{s}^{2} v\left(x_{s}\right)+2 c_{s} d_{s} w\left(x_{s}\right)\right\}\right] \\
& =C^{\prime} A C>0 .
\end{aligned}
$$

The positive definiteness of the matrix $A$ follows from the fact that $g(x) v(x)-w^{2}(x)=$ $g^{2}(x) \operatorname{Var}[Y \mid X=x]$ and $g\left(x_{i}\right)>0$ for $i=1,2$. It now suffices to prove that

$$
\lim _{n \rightarrow \infty} n^{-3 / 2}\left\{\sum_{i=1}^{n} E\left|C^{\prime} W_{i}\right|^{3}\right\}=0
$$

Using the hypothesis and the arguments similar to those in Lemma 1 it can be shown that

$$
E\left|U_{i}\left(x_{s}\right)\right|^{3}=O\left(h_{n}^{3 / 2} h_{i}^{-2}\right)=O\left(h_{n}^{-1 / 2}\right)
$$

and

$$
E\left|V_{i}\left(x_{s}\right)\right|^{3}=O\left(h_{n}^{3 / 2} h_{i}^{-3}\right)=O\left(h_{n}^{-3 / 2}\right)
$$

Hence 


$$
\begin{aligned}
n^{-3 / 2}\left\{\sum_{i=1}^{n} E\left|C^{\prime} W_{i}\right|^{3}\right\} & \leqq n^{-3 / 2}|C|^{3} \sum_{i=1}^{n} E\left|W_{i}\right|^{3} \\
& \leqq 8 n^{-3 / 2}|C|^{3} \sum_{i=1}^{n} \max _{s=1,2}\left\{E\left|U_{i}\left(x_{s}\right)\right|^{3}, E\left|V_{i}\left(x_{s}\right)\right|^{3}\right\} \\
& =O\left\{\left(n h_{n}^{3}\right)^{-1 / 2}\right\}=o(1) .
\end{aligned}
$$

This completes the proof.

We define

$$
\begin{aligned}
Z_{n}^{*}= & h_{n}^{1 / 2} \cdot n^{-1 / 2}\left\{\sum_{i=1}^{n}\left(U_{i}^{*}\left(x_{1}\right)-g\left(x_{1}\right)\right), \sum_{i=1}^{n}\left(V_{i}^{*}\left(x_{1}\right)-w\left(x_{1}\right)\right),\right. \\
& \left.\sum_{i=1}^{n}\left(U_{i}^{*}\left(x_{2}\right)-g\left(x_{2}\right)\right), \sum_{i=1}^{n}\left(V_{i}^{*}\left(x_{2}\right)-w\left(x_{2}\right)\right)\right\} .
\end{aligned}
$$

Lemma 3. Suppose conditions (i) through (vi) are satisfied. Let $E_{f}|Y|^{3}$ be finite and let $g^{\prime}, g^{\prime \prime}, w^{\prime}, w^{\prime \prime}$ and $v^{\prime}$ exist and be bounded. If $g\left(x_{i}\right)>0$ for $i=1,2$, then $C^{\prime} Z_{n}^{*}$ converges in distribution to a normal random variable with mean 0 and variance $C^{\prime} A C$.

Proof. We have

$$
C^{\prime} Z_{n}-C^{\prime} Z_{n}^{*}=h_{n}^{1 / 2} \cdot n^{-1 / 2} \sum_{i=1}^{n} \sum_{s=1}^{2}\left[c_{s}\left\{g\left(x_{s}\right)-E U_{i}^{*}\left(x_{s}\right)\right\}+d_{s}\left\{w\left(x_{s}\right)-E V_{i}^{*}\left(x_{s}\right)\right\}\right] .
$$

Using the hypothesis it can be shown that for $i=1,2, \cdots, n$ and $s=1,2$

and

$$
E U_{i}^{*}\left(x_{s}\right)=g\left(x_{s}\right)+O\left(h_{i}^{2}\right)
$$

$$
E V_{i}^{*}\left(x_{s}\right)=w\left(x_{s}\right)+O\left(h_{i}^{2}\right) \text {. }
$$

Hence,

$$
\left|\sum_{i=1}^{n}\left(g\left(x_{s}\right)-E U_{i}^{*}\left(x_{s}\right)\right)\right|=O\left(\sum_{i=1}^{n} h_{i}^{2}\right)
$$

and

$$
\left|\sum_{i=1}^{n}\left(w\left(x_{s}\right)-E V_{i}^{*}\left(x_{s}\right)\right)\right|=O\left(\sum_{i=1}^{n} h_{i}^{2}\right) .
$$

From (8), (9) and (10) we get

$$
\begin{aligned}
C^{\prime} Z_{n}-C^{\prime} Z_{n}^{*} & =O\left(h_{n}^{1 / 2} n^{-1 / 2} \sum_{i=1}^{n} h_{i}^{2}\right) \\
& =O\left(\left(n h_{n}\right)^{1 / 2} \frac{1}{n} \sum_{i=1}^{n} h_{i}^{2}\right) \\
& =O\left(\left(n h_{n}\right)^{1 / 2} h_{n}^{2}\right) \\
& =O\left(\left(n h_{n}^{5}\right)^{1 / 2}\right) \\
& =O(1) .
\end{aligned}
$$

The proof now follows from Lemma 2.

Suppose $N_{t}(t>0)$ is a stopping random variable such that $\frac{N_{t}}{t} \stackrel{p}{\rightarrow} \pi(\pi>0)$ as $t \rightarrow \infty$. This implies that for any $\varepsilon>0$, there exists $t_{0}=t_{0}(\varepsilon)$ such that for $t \geqq t_{0}$ we have 
We define

$$
P\left[\left|N_{t}-\pi t\right| \geqq \pi t \varepsilon\right]<\varepsilon .
$$

$$
N_{1}=N_{1}(t, \varepsilon)=[\pi t(1-\varepsilon)]
$$

and

$$
N_{2}=N_{2}(t, \varepsilon)=[\pi t(1+\varepsilon)]
$$

where $[x]$ is the integral part of $x$.

We note that for any $0<\varepsilon<\frac{1}{2}$ and $t>\frac{1}{\pi \varepsilon}$ the numbers $N_{1}$ and $N_{2}$ defined above satisfy the following inequalities:

$$
\frac{N_{2}}{N_{1}}<\frac{1+\varepsilon}{1-2 \varepsilon}
$$

and

We define

$$
\frac{N_{2}-N_{1}}{N_{1}}<\frac{3 \varepsilon}{1-2 \varepsilon}
$$

$$
\begin{aligned}
& R_{i}=\sum_{s=1}^{2}\left\{c_{s} U_{i}^{*}\left(x_{s}\right)+d_{s} V_{i}^{*}\left(x_{s}\right)\right\}, \quad i=1,2, \cdots \\
& \mu=\sum_{s=1}^{2}\left\{c_{s} g\left(x_{s}\right)+d_{s} w\left(x_{s}\right)\right\} \\
& S_{n}=\sum_{i=1}^{n}\left\{R_{i}-\mu\right\}, \quad n=1,2, \cdots \\
& Q=\max _{N_{1}<n \leq N_{2}}\left|\sum_{i=N_{1}+1}^{n}\left[R_{i}-E\left\{R_{i}\right\}\right]\right| .
\end{aligned}
$$

It can be seen that $h_{n}^{1 / 2} n^{-1 / 2} S_{n}=C^{\prime} Z_{n}^{*}$. Replacing $n$ by $N_{t}$ in the expressions for $\bar{U}_{n}\left(x_{s}\right)$, $\bar{V}_{n}\left(x_{s}\right), Z_{n}$ and $Z_{n}^{*}$, we define $\bar{U}_{N_{t}}\left(x_{s}\right), \bar{V}_{N_{t}}\left(x_{s}\right), Z_{N_{t}}$ and $Z_{N_{t}}^{*}$ respectively.

In order to study the asymptotic distribution of $C^{\prime} Z_{N_{t}}^{*}$ we find it convenient to choose a specific sequence $\left\{h_{n}=n^{-\delta}, n=1,2, \cdots\right\}$, where $\delta$ is some positive number. With this choice of $\left\{h_{n}\right\}$ we have the following lemma. Let $C_{1}$ be a generic constant.

LEMMA 4. Suppose $K$ satisfies conditions (i) and (iii) and $\left\{h_{n}=n^{-\delta}\right\}, \delta>0$. If $g^{\prime}$ and $v^{\prime}$ exist and are bounded, then for any $0<\varepsilon<\frac{1}{2}, t>\frac{1}{\pi \varepsilon}$ we have

$$
P\left\{Q \geqq \varepsilon^{1 / 3} \sqrt{\frac{N_{1}}{h_{N_{1}}}}\right\}<\frac{C_{1} \varepsilon^{1 / 3}}{(1-2 \varepsilon)}\left\{\frac{(1+\varepsilon)}{(1-2 \varepsilon)}\right\}^{\delta}
$$

Proof. By Kolmogorov's inequality

$$
P\left[Q \geqq \varepsilon^{1 / 3} \sqrt{\frac{N_{1}}{h_{N_{1}}}}\right] \leqq \frac{\sum_{i=N_{1}+1}^{N_{2}} E\left\{R_{i}^{2}\right\}}{\varepsilon^{2 / 3}\left\{-N_{1}\right\}} .
$$

Using the hypothesis it can be shown that

$$
E\left\{R_{i}^{2}\right\}=O\left(\frac{1}{h_{i}}\right)=O\left(\frac{1}{h_{N_{2}}}\right) \quad \text { if } \quad N_{1}<i \leqq N_{2} .
$$


From (13) and (14), we get

$$
\begin{aligned}
P\left[Q \geqq \varepsilon^{1 / 3} \sqrt{\frac{N_{1}}{h_{N_{1}}}}\right] & \leqq \frac{C_{1}\left(N_{2}-N_{1}\right)}{3 \varepsilon^{2 / 3} N_{1}}\left(\frac{h_{N_{1}}}{h_{N_{2}}}\right) \\
& \leqq \frac{C_{1} \varepsilon^{1 / 3}}{(1-2 \varepsilon)}\left(\frac{1+\varepsilon}{(1-2 \varepsilon)}\right)^{\delta} .
\end{aligned}
$$

Lemma 5. Suppose $K$ satisfies conditions (ii), and (iii) and $\left\{h_{n}=n^{-\delta}\right\}, \delta>0$. If $g^{\prime}, g^{\prime \prime}$, $w^{\prime}$ and $w^{\prime \prime}$ exist and are bounded and if $0<\varepsilon<\frac{1}{2}, t>\frac{1}{\pi \varepsilon}$ and $\frac{1}{5}<\delta$, then for all $N_{1}<n$ $\leqq N_{2}$,

$$
\left|\sqrt{\frac{h_{N_{1}}}{N_{1}}} \sum_{i=N_{1}+1}^{n}\left[E\left\{R_{i}\right\}-\mu\right]\right|<\frac{C_{1} \varepsilon}{(1-2 \varepsilon)} .
$$

PROOF. Using computations similar to those in Lemma 3, we obtain for all $n$ such that $N_{1}<n \leqq N_{2}$

$$
\begin{aligned}
\left|\sum_{i=N_{1}+1}^{n}\left[E\left\{R_{i}\right\}-\mu\right]\right| & \leqq \frac{C_{1}}{3}\left(N_{2}-N_{1}\right) \sum_{i=N_{1}+1}^{n} h_{i}^{2} \\
& \leqq \frac{C_{1}}{3}\left(N_{2}-N_{1}\right) h_{N_{1}}^{2} .
\end{aligned}
$$

Hence,

$$
\begin{aligned}
\left|\sqrt{\frac{h_{N_{1}}}{N_{1}}} \sum_{i=N_{1}+1}^{n}\left[E\left\{R_{i}\right\}-\mu\right]\right| & \leqq \frac{C_{1}\left(N_{2}-N_{1}\right)}{3 N_{1}}\left(N_{1} h_{N_{1}}^{5}\right)^{1 / 2} \\
& =\frac{C_{1}\left(N_{2}-N_{1}\right)}{3 N_{1}}\left(N_{1}^{1-5 \delta}\right)^{1 / 2} \\
& \leqq \frac{C_{1} \varepsilon}{(1-2 \varepsilon)}
\end{aligned}
$$

Lemma 6. Suppose $K$ satisfies conditions (i), (ii) and (iii) and $\left\{h_{n}=n^{-\delta}\right\}, \frac{1}{5}<\delta<\frac{1}{3}$ and $\frac{N_{t}}{t} \stackrel{p}{\rightarrow} \pi(\pi>0)$ as $t \rightarrow \infty$. Let $E_{f}|Y|^{3}$ be finite and let $g^{\prime}, g^{\prime \prime}, w^{\prime}, w^{\prime \prime}$ and $v^{\prime}$ exist and be bounded. If $x_{1} \neq x_{2}, g\left(x_{i}\right)>0$ for $i=1,2$, then $C^{\prime} Z_{N_{t}}^{*}$ converges in distribution to a normal random variable with mean 0 and variance $C^{\prime} A C$ as $t$ tends to infinity.

Proof. The proof resembles that of Theorem 1 in Renyi [5]. Let $\varepsilon\left(\varepsilon<\frac{1}{2}\right)$ be an arbitrarily small positive number. Let $t \geqq t_{0}$ where $t_{0}=t_{0}(\varepsilon)>\frac{1}{\pi \varepsilon}$ and let $N_{1}$ and $N_{2}$ be chosen as before.

We have for $y>0$

$$
\begin{aligned}
P\left[C^{\prime} Z_{N_{t}}^{*}<y\right]= & \sum_{n=1}^{\infty} P\left[C^{\prime} Z_{n}^{*}<y ; N_{t}=n\right] \\
= & \sum_{\mid n-\pi t i<\pi t \varepsilon} P\left[C^{\prime} Z_{n}^{*}<y ; N_{t}=n\right] \\
& +\sum_{|n-\pi t| \geq \pi t \varepsilon} P\left[C^{\prime} Z_{n}^{*}<y ; N_{t}=n\right] \\
\leqq & \sum_{|n-\pi|<\pi t \varepsilon} P\left[C^{\prime} Z_{n}^{*}<y ; N_{t}=n\right]+\varepsilon .
\end{aligned}
$$


Hence,

$$
\left|P\left[C^{\prime} Z_{N_{t}}^{*}<y\right]-\sum_{\mid n-\pi i t_{i \pi t \varepsilon}} P\left[C^{\prime} Z_{n}^{*}<y ; N_{t}=n\right]\right|<\varepsilon .
$$

Introducing the random variables $R_{i}, S_{n}$ and $Q$ as defined in (11), we have for any $n$ such that $N_{1}<n \leqq N_{2}$

$$
\begin{aligned}
P\left[C^{\prime} Z_{n}^{*}<y ; N_{t}=n\right]= & P\left[\sqrt { \frac { h _ { n } } { n } } \left\{\sum_{i=1}^{N_{1}}\left(R_{i}-\mu\right)+\sum_{i=N_{1}+1}^{n}\left(R_{i}-E\left(R_{i}\right)\right)\right.\right. \\
& \left.\left.+\sum_{i=N_{1}+1}^{n}\left(E\left(R_{i}\right)-\mu\right)\right\}<y ; N_{t}=n\right] \\
\leqq & P\left[S_{N_{1}}<y \sqrt{\frac{N_{2}}{h_{N_{2}}}}-\sum_{i=N_{1}+1}^{n}\left(E\left(R_{i}\right)-\mu\right)+Q ; N_{t}=n\right] \\
= & P\left[\sqrt{\frac{h_{N_{1}}}{N_{1}}} S_{N_{1}}<y \sqrt{\frac{N_{2}}{N_{1}}\left(\frac{h_{N_{1}}}{h_{N_{2}}}\right)}-\sqrt{\frac{h_{N_{1}}}{N_{1}}} \sum_{i=N_{1}+1}^{n}\left\{E\left(R_{i}\right)-\mu\right\}\right. \\
& \left.+\sqrt{\frac{h_{N_{1}}}{N_{1}}} Q ; N_{t}=n\right] \\
\leqq & P\left[C^{\prime} Z_{N_{1}}^{*}<y \sqrt{\left(\frac{N_{2}}{N_{1}}\right)^{1+\delta}}+\frac{C_{1} \varepsilon}{(1-2 \varepsilon)}+\sqrt{\frac{h_{N_{1}}}{N_{1}}} Q ; N_{t}=n\right],
\end{aligned}
$$

by Lemma 5 . Hence,

$$
\begin{aligned}
& \sum_{|n-\pi t|<\pi t \varepsilon} P\left[C^{\prime} Z_{n}^{*}<y ; N_{t}=n\right] \\
& \leqq P\left[C^{\prime} Z_{N_{1}}^{*}<y \sqrt{\left(\frac{N_{2}}{N_{1}}\right)^{1+\delta}}+\frac{C_{1} \varepsilon}{(1-2 \varepsilon)}+\sqrt{\frac{h_{N_{1}}}{N_{1}}} Q ;\left|N_{t}-\pi t\right|<\pi t \varepsilon\right] \\
& \leqq P\left[C^{\prime} Z_{N_{1}}^{*}<y\left(\frac{1+\varepsilon}{(1-2 \varepsilon)}\right)^{(1+\delta) / 2}+\frac{C_{1} \varepsilon}{(1-2 \varepsilon)}+\varepsilon^{1 / 3}\right. \text {; } \\
& \left.Q<\varepsilon^{1 / 3} \sqrt{\frac{N_{1}}{h_{N_{1}}}} ;\left|N_{t}-\pi t\right|<\pi t \varepsilon\right]+P\left[Q \geqq \varepsilon^{1 / 3} \sqrt{\frac{N_{1}}{h_{N_{1}}}}\right] \\
& \leqq P\left[C^{\prime} Z_{N_{1}}^{*}<y\left(\frac{1+\varepsilon}{1-2 \varepsilon}\right)^{(1+\delta) / 2}+\frac{C_{1} \varepsilon}{(1-2 \varepsilon)}+\varepsilon^{1 / 3}\right]+\frac{C_{1} \varepsilon^{1 / 3}}{(1-2 \varepsilon)}\left(\frac{1+\varepsilon}{1-2 \varepsilon}\right)^{\delta},
\end{aligned}
$$

by Lemma 4 .

From (16) we get in a similar manner

$$
\begin{aligned}
& \sum_{\mid n-\pi t i<\pi t \varepsilon} P\left[C^{\prime} Z_{n}^{*}<y ; N_{t}=n\right] \\
& \quad \geqq P\left[C^{\prime} Z_{N_{1}}^{*}<y-\frac{C_{1} \varepsilon}{(1-2 \varepsilon)}-\sqrt{\frac{h_{N_{1}}}{N_{1}}} Q ;\left|N_{t}-\pi t\right|<\pi t \varepsilon\right] \\
& \quad \geqq P\left[C^{\prime} Z_{N_{1}}^{*}<y-\frac{C_{1} \varepsilon}{(1-2 \varepsilon)}-\varepsilon^{1 / 3} ; Q<\varepsilon^{1 / 3} \sqrt{\frac{N_{1}}{h_{N_{1}}}} ;\left|N_{t}-\pi t\right|<\pi t \varepsilon\right] \\
& \quad \geqq P\left[C^{\prime} Z_{N_{1}}^{*}<y-\frac{C_{1} \varepsilon}{(1-2 \varepsilon)}-\varepsilon^{1 / 3}\right]-P\left[Q \geqq \varepsilon^{1 / 3} \sqrt{\frac{N_{1}}{h_{N_{1}}}}\right]-P\left[\left|N_{t}-\pi t\right| \geqq \pi t \varepsilon\right] \\
& \quad \geqq P\left[C^{\prime} Z_{N_{1}}^{*}<y-\frac{C_{1} \varepsilon}{(1-2 \varepsilon)}-\varepsilon^{1 / 3}\right]-\frac{C_{1} \varepsilon^{1 / 3}}{(1-2 \varepsilon)}\left(\frac{1+\varepsilon}{1-2 \varepsilon}\right)^{\delta}-\varepsilon .
\end{aligned}
$$


From (15), (17) and (18) we conclude that for $t \geqq t_{0}$

and

$$
P\left[C^{\prime} Z_{N_{t}}^{*}<y\right] \leqq P\left[C^{\prime} Z_{N_{1}}^{*}<y\left(\frac{1+\varepsilon}{1-2 \varepsilon}\right)^{(1+\delta) / 2}+\frac{C_{1} \varepsilon}{(1-2 \varepsilon)}+\varepsilon^{1 / 3}\right] \div \frac{C_{1} \varepsilon^{1 / 3}}{(1-2 \varepsilon)}\left(\frac{1+\varepsilon}{1-2 \varepsilon}\right)^{\delta}+\varepsilon
$$

$$
P\left[C^{\prime} Z_{N_{t}}^{*}<y\right] \geqq P\left[C^{\prime} Z_{N_{1}}^{*}<y-\frac{C_{1} \varepsilon}{(1-2 \varepsilon)}-\varepsilon^{1 / 3}\right]-\frac{C_{1} \varepsilon^{1 / 3}}{(1-2 \varepsilon)}\left(\frac{1+\varepsilon}{1-2 \varepsilon}\right)^{\delta}-2 \varepsilon .
$$

Similar statements hold for $y<0$. We now invoke Lemma 3 and the continuity of the distribution function of a normal random variable to complete the proof.

We are now in a position to prove the main theorem of this paper.

THEOREM. Suppose $K$ satisfies conditions (i), (ii) and (iii), and $\left\{h_{n}=n^{-o}\right\}, \frac{1}{5}<\delta<\frac{1}{3}$ and $\frac{N_{t}}{t} \rightarrow \pi(\pi>0)$ as $t \rightarrow \infty$. Let $E_{f}|Y|^{3}$ be finite and let $g^{\prime}, g^{\prime \prime}, w^{\prime}, w^{\prime \prime}$ and $v^{\prime}$ exist and be bounded. If $x_{1} \neq x_{2}, g\left(x_{i}\right)>0$ for $i=1,2$, then $\left(N_{t} h_{N_{t}}\right)^{1 / 2}\left(m_{N_{t}}\left(x_{1}\right)-m\left(x_{1}\right), m_{N_{t}}\left(x_{2}\right)\right.$ $\left.-m\left(x_{2}\right)\right)^{\prime}$ converges in distribution to $Z^{*}$ as $t$ tends to infinity where $Z^{*}$ is a bivariate normal random variable with mean vector 0 and diagonal covariance matrix $C=\left[c_{i j}\right]$ where

$$
c_{i i}=\frac{\operatorname{Var}\left[Y \mid X=x_{i}\right]}{g\left(x_{i}\right)} \nu \int_{-\infty}^{\infty} K^{2}(u) d u \quad i=1,2 .
$$

PRoof. Using the Cramér-Wold theorem (Theorem (xi) on page 123 of [4]) we conclude from Lemma 6 that $Z_{N_{t}}^{*}$ converges in distribution to $Z$ as $t$ tends to infinity. The proof of the theorem now immediately follows from this result in conjunction with Theorem (iii) on page 388 of [4].

\section{References}

[1] Korovkin, P.P.: Inequalities. Pergammon Press, London, (1961).

[2] Loeve, M.: Probability Theory. Third Edition, Van Nostrand, Princeton, (1963).

[3] Nadaraya, E. A. : “On Estimating Regression". Theory of Probability and Its Applications, 9 (1964), 141-142.

[4] RAO, C.R.: Linear Statistical Inference and Its Applications. Second Edition, Wiley, New York, (1973).

[5] RENYI, A.: On the Asymptotic Distribution of the Sum of a Random Number of Independent Random Variables. Acta Mathematica Scientiarum Hungaricae, 8 (1957), 193-199.

[6] Schuster, E.F.: Joint Asymptotic Distribution of the Estimated Regression at a Finite Number of Distinct Points. Annals of Mathematical Statistics, 43 (1972), 84-88.

[7] Watson, G. S.: “Smooth Regression Analysis”. Sankhyá, A26 (1964), 359-372.

[8] Yamato, H.: "Sequential Estimation of a Continuous Probability Density Function and Mode". Bulletin of Mathematical Statistics, 14, 3-4, (1971), 1-12.

Communicated by S. Kano

Received June 20, 1983 